This is the peer reviewed version of the following article:

Austen, S. and Jefferson, T. and Lewin, G. and Ong, R. and Sharp, R. 2015. Work ability, age and intention to leave aged care work. Australasian Journal on Ageing. 35 (1): pp. 18-22.,

which has been published in final form at http://doi.org/10.1111/ajag.12187

This article may be used for non-commercial purposes in accordance with Wiley Terms and Conditions for Self-Archiving at http://olabout.wiley.com/WileyCDA/Section/id-

820227.html\#terms 


\section{Work ability, age and intention to leave aged care work}

Siobhan Austen

School of Economics and Finance, Curtin University, Perth, Western Australia, Australia

Therese Jefferson

Curtin Graduate School of Business, Curtin University, Perth, Western Australia, Australia

\section{Gill Lewin}

Centre for Research on Ageing, Curtin University; and Silver Chain, Perth, Western Australia, Australia

\section{Rachel Ong}

School of Economics and Finance, Curtin University, Perth, Western Australia, Australia

\section{Rhonda Sharp}

Hawke Research Institute, University of South Australia, Adelaide, South Australia, Australia

Aim: To describe the work ability of mature age women workers in Australia's aged care sector, and to explore the relationship between ageing, work ability and intention to leave.

Method: Logistic regression techniques were applied to a sample of 2721 responses to a survey of mature age women workers in the aged care sector.

Results: Mature age women working in the aged care sector have relatively high levels of work ability by international standards. Furthermore, their work ability remains high in their 50 s and 60 s, in contrast to some prevailing stereotypes. However, work ability is a key determinant of intention to leave in key occupational groups.

Conclusion: Our findings challenge some prevailing stereotypes about the work ability of mature age workers. However, they lend support for the development of retention strategies, which incorporate programmes that target low work ability.

Key words: intention to leave, quantitative method, work ability.

\section{Introduction}

Work ability is a complex concept that refers to workers' ability at present and in the near future to meet the demands of their jobs. Brook et al. [1] demonstrated the relevance of the concept to the Australian aged care workforce in their study of 64 personal care assistants (PCAs) and identified the

Correspondence to: Associate Professor Siobhan Austen, School of Economics and Finance, Curtin University. Tel: +61 08 92667343; Fax:+61 08 92663026; Email: siobhan.austen@cbs.curtin.edu.au effectiveness of several actions to improve the work ability of these workers. The current study is larger and broader. It investigates the work ability of mature age women workers in Australia's aged care sector, exploring the relationship between ageing, work ability and the intention to leave (ITL) aged care work. The study is based on the responses of 2721 Australian aged care workers to a survey that we conducted in 2011, making it the largest single-country study of the work ability of mature age women workers in aged care.

The link between work ability, ageing and ITL for mature age women workers in the aged care sector has strategic importance. Women comprise more than $90 \%$ of the Australian aged care workforce and the median age of this workforce is already close to 50 [2]. The Productivity Commission predicts that the number of older Australians needing aged care will more than triple from around 1 million to 3.5 million in 2050, necessitating a quadrupling of the aged care workforce by the same year [3]. While the retention of mature age women workers will be critical to the community's capacity to provide for its future care needs [4], existing research that specifically addresses this cohort is limited $[5,6]$.

\section{Methods}

\section{Data collection}

We collected survey data from women aged 45 or over who were working in a direct care role in aged care in November 2011. A paper survey was sent first to 18 aged care service providers who then posted it to the homes of their employees in the relevant age group and roles. Six thousand eight hundred sixty-seven mature age women workers in the aged care sector were sent the survey, covering all the key direct care roles (registered nurses (RNs), enrolled nurses (ENs), PCAs and community care workers (CCW) ). Participation in the survey was voluntary. Participants returned the survey in prepaid envelopes to the research team. ${ }^{1}$

The aged care providers involved in the study were all members of Aged and Community Services Australia (ACSA). They spanned all the Australian states (with two each in New South Wales, Victoria and Queensland; three each in Tasmania and South Australia; and seven in Western Australia) and included urban-based, as well as rural-based, providers.

${ }^{1}$ The research design, including the data collection, access and storage processes, was approved by the Human Research Ethics Committee at Curtin University (approval number HR18/2011). The survey was also made available online and on the website of the Australian Nurses' Federation. 
Table 1: Sample socio-demographics by occupation (Australian female aged care workers aged 45+, 2011)

\begin{tabular}{|c|c|c|c|c|c|c|}
\hline & \multirow[t]{2}{*}{$n$} & \multicolumn{3}{|c|}{ Age $(\%)$} & \multicolumn{2}{|c|}{ Workplace (\%) } \\
\hline & & $45-49$ & $50-54$ & $\overline{55+}$ & Residential & Home care \\
\hline Registered nurses & 533 & 15.4 & 28.1 & 56.5 & 78.6 & 21.4 \\
\hline Enrolled nurses & 428 & 21.3 & 30.6 & 48.1 & 91.9 & 8.1 \\
\hline Personal care assistants & 1149 & 22.8 & 28.3 & 48.9 & 77.1 & 22.9 \\
\hline Community care workers & 611 & 20.6 & 23.7 & 55.6 & 89 & 91.1 \\
\hline Total & 2721 & 20.6 & 27.6 & 51.8 & 64.2 & 35.8 \\
\hline
\end{tabular}

In total (across the online and paper versions of the survey), 3945 respondents completed the survey. Two thousand eight hundred fifty respondents completed the paper version of the survey (representing a response rate of $41.5 \%$ ). The sample used in the current analysis of work ability is smaller at 2721 as it excludes (i) the survey respondents who could not be reliably classified into one of the key occupational roles; and (ii) the respondents who did not provide valid responses to each of the survey questions relevant to the measurement of work ability. However, the sample of mature age women workers involved in the aged care sector is still large by international standards. In Camerino et al.'s [7] study of work ability, the largest country sample of mature age nurses was 463, in the Netherlands. Ilmarinen et al.'s [8] Finnish work ability study included 94 mature age nurses and 56 mature age home care workers.

\section{Measures}

To measure work ability we included seven items in the survey to probe the workers' health, as well as their subjective estimation of their resources in relation to work demands. The items targeted the workers' (i) subjective estimation of present work ability compared with lifetime best performance; (ii) subjective work ability in relation to both physical and mental demand of the work; (iii) number of diagnosed diseases (from a list of 15); (iv) subjective estimation of working impairment due to ill health; (v) sickness absence during the past year; (vi) own prognosis of work ability after two years; and (vii) psychological resources (enjoying daily tasks, activity and life spirit, optimistic about the future). We used the items to create a Work Ability Index (WAI) score for each worker. As summarised by van den Berg et al. [9], a large number of studies over the last quarter century have measured work ability using this index. Following these studies, we created a WAI score for each worker by allocating points to each item, producing a summative score that ranged from 7 to 49 points. In our analysis of the links between work ability and ITL, we followed a study of the work ability of European nurses conducted by Camerino et al. [7] and first transformed the raw WAI scores into Z-scores to identify three categories of work ability: (i) 'Low WAI' (where $\mathrm{z}$ " $\square 1$ ); (ii) 'medium WAI' (where $\square 1 " \mathrm{z} " 1$ ); and (iii) 'high' WAI' (where $\mathrm{z} \geq 1$ ).

ITL is measured to gauge a worker's likely future retention in her job. In our study, ITL was measured by a survey question that asked, first, 'In the past year have you ever thought about leaving the aged care sector?' A follow-up question asked: 'How often do you have this thought?' The possible responses were 'At least once a year', 'At least once a month', 'At least once a week' and 'Every day'. We grouped these responses into two categories: (i) 'Low ITL', where the response indicated that the person had not thought about leaving or had thought about leaving only once in the past year; and (ii) 'High ITL', where the response indicated the person had thought about leaving at least once a month, week or day.

\section{Analysis}

In this paper we examine the differences in work ability across the occupational groups of mature age women workers in Australia's aged care sector before assessing the association between WAI scores and age in each occupational group. Multiple logistic regression analyses are then performed to examine the association between work ability and ITL in the occupational groups, controlling for age and type of workplace (residential aged care facilities and community settings). ${ }^{2}$

\section{Results}

The socio-demographic characteristics of our sample are summarised in Table 1. These data show that PCAs are the largest occupational group, comprising $42.5 \%$ of the sample. These workers typically provide personal care and support to clients in residential aged care facilities. Together, RNs and ENs account for $35.3 \%$ of the sample. CCWs comprise $22.2 \%$ of the sample. ${ }^{3}$ They typically provide personal care and support to older people living at home.

\section{Work ability and type of work}

The data in Table 2 summarise the work ability of mature age women in each occupational group. The data indicate that, compared with the other groups of nurses and care workers in aged care, RNs achieve, on average, a relatively low level of measured work ability. Twenty point eight per cent of RNs are classified as having low work ability overall, as compared with $9.7 \%$ of CCWs, $15.7 \%$ of ENs and $13.8 \%$ of PCAs.

${ }^{2}$ SPSS Version 20 is used for data handling.

${ }^{3}$ A larger survey of the Australian aged care work force by Martin and King [10] identified a similar occupational distribution $(45.4 \%$ PCAs, $22.3 \%$ nurses and $32.3 \%$ Community Care Workers). 
Table 2: Work ability scores on the Work Ability Index (WAI), by occupation (Australian female aged care workers aged $45+, 2012)$

\begin{tabular}{lcccr}
\hline & $\begin{array}{c}\text { Registered } \\
\text { nurses }\end{array}$ & $\begin{array}{c}\text { Enrolled } \\
\text { nurses }\end{array}$ & $\begin{array}{c}\text { Personal care } \\
\text { assistants }\end{array}$ & $\begin{array}{c}\text { Community care } \\
\text { workers }\end{array}$ \\
\hline \% Low WAl & 20.8 & 15.7 & 13.8 & 9.7 \\
\% Medium WAl & 68.1 & 74.8 & 71.5 & 70.9 \\
\% High WAl & 11.1 & 9.6 & 14.7 & 19.5 \\
\hline
\end{tabular}

Table 3: Rates of high ITL by total Work Ability Index (WAI) score and occupation (Australian female aged care workers aged 45+, 2012)

\begin{tabular}{|c|c|c|c|c|c|}
\hline & $n$ & $\begin{array}{c}\text { Low WAI } \\
(\%)\end{array}$ & $\underset{(\%)}{\text { Medium WAI }}$ & $\begin{array}{c}\text { High WAI } \\
(\%)\end{array}$ & $\begin{array}{c}\text { Chi-squared } \dagger \\
\quad(P \text { value })\end{array}$ \\
\hline Registered nurses & 531 & 16.2 & 19.1 & 10.2 & 2.97 \\
\hline Enrolled nurses & 425 & 28.8 & 24.1 & 10.0 & 5.15 \\
\hline Personal care assistants & 1135 & 41.0 & 19.4 & 16.3 & $39.28^{\star * *}$ \\
\hline Community care workers & 606 & 46.6 & 17.4 & 9.3 & $36.95^{\star * *}$ \\
\hline Total & 2697 & 32.7 & 19.7 & 12.5 & $51.42^{* * *}$ \\
\hline
\end{tabular}

${ }^{* *} P<0.001 . \dagger$ d. $f=2$ for each occupational group.

\section{Work ability and age}

Our data also indicate differences in work ability associated with age. A statistically significant negative relationship between age and perceived work ability was observed in the overall sample (ANCOVA F-test, adjusted for occupational group: $\left.\mathrm{F}_{(2,2715)}=3.222, P=0.040\right)$. However, it is important to note that this relationship is largely driven by a $5 \%$ difference between the work ability score of RNs aged 45-49 and those aged 50-54. As shown in Figure 1, only marginal changes in the average work ability of ENs, PCAs and CCWs are associated with differences in age. When the data for the groups of nurses and care workers are analysed separately, the relationship between age and perceived work ability is not statistically significant.

\section{Work ability and ITL}

Our study finds evidence of a negative impact of work ability on ITL. Across the sample, ITL was higher among those with low work ability. As shown in Table 3, 32.7\% of the sample with low work ability reported that they thought about leaving each day, week or month. Among the workers with high work ability this rate was only $12.5 \%$.

However, the data in Table 3 also show that the relationship between work ability and ITL differs between the groups of nurses and care workers. It is strongest among PCAs and CCWs. Close to half $(46.6 \%)$ of the CCWs with low work ability have a high ITL, whereas only $9.3 \%$ of CCWs with high work ability have a high ITL. Among RNs, on the other hand, this relativity is $16.2 \%$ and $10.2 \%$.

More precise data on the relationship between work ability and ITL are presented in Table 4. These include the odds ratios and $95 \%$ confidence intervals obtained by logistic regression analyses, adjusted for age and workplace (in the
Figure 1: Association between age and work ability (Work Ability Index score) by occupation (Australian female aged care workers aged 45+, 2012).

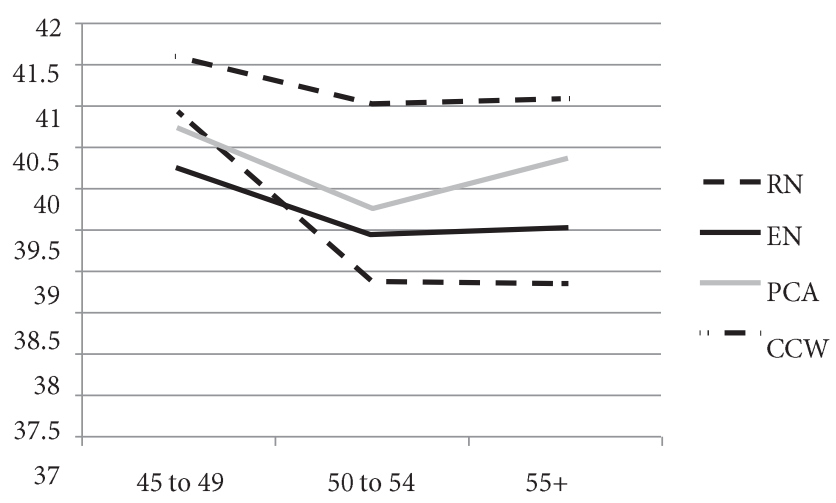

case of nurses ${ }^{4}$ ), of the relationship between the likelihood of 'high ITL' and high versus low and medium work ability.

The data in Table 4 indicate that, across the sample, nurses and care workers with medium work ability are, on average, 1.8 times more likely to have a high ITL than their counterparts with high work ability. Mature age nurses and care workers with low work ability are, on average, 4.1 times more likely to have a high ITL than their counterparts with high work ability. The data in the table also confirm that the relationship between work ability and ITL is strongest among the CCWs and weakest for RNs. CCWs with low work ability are 8.5 times more likely to have a high ITL than

${ }^{4}$ Controls for workplace are not included for CCWs and PCAs as very few of the former are employed in residential workplaces and very few of the latter are employed in community (home care) settings. 
Austen S, Jefferson T, Lewin G et al.

Table 4: Association between total Work Ability Index (WAI) score and ITL $\dagger$ by occupation (Australian female aged care workers aged $45+$, 2012)

\begin{tabular}{|c|c|c|c|c|c|c|}
\hline \multirow{2}{*}{ Registered nurses } & \multirow{2}{*}{$\begin{array}{c}\begin{array}{c}\text { Odds ratio } \\
\text { medium WAlf }\end{array} \\
1.81\end{array}$} & \multicolumn{2}{|c|}{$95 \% \mathrm{Cl}$} & \multirow{2}{*}{$\begin{array}{c}\text { Odds ratio } \\
\text { low WAlf } \\
1.66\end{array}$} & \multicolumn{2}{|c|}{$95 \% \mathrm{Cl}$} \\
\hline & & 0.73 & 4.49 & & 0.60 & 4.64 \\
\hline Enrolled nurses & $3.57^{\star \star}$ & 1.06 & 12.00 & $4.41^{* *}$ & 1.19 & 16.27 \\
\hline Personal care assistants & 1.22 & 0.78 & 1.91 & $3.54^{\star \star \star}$ & 2.10 & 5.97 \\
\hline Community care workers & $2.06^{* *}$ & 1.05 & 4.02 & $8.50^{* * *}$ & 3.79 & 19.07 \\
\hline Total & $1.83^{\star * *}$ & 1.35 & 2.48 & $4.09^{* \star *}$ & 2.89 & 5.80 \\
\hline
\end{tabular}

†The reference category is low ITL. $\ddagger$ The reference category is high WAl. ${ }^{* *} P<0.05 ;{ }^{* \star *} P<0.001$.

their counterparts with high work ability. However, there is not a statistically significant difference in the odds of high ITL between RNs with a high versus low work ability.

\section{Discussion}

This study finds that, compared with other mature age nurses and care workers in the Australian aged care sector, RNs achieve relatively poor levels of work ability. However, when the comparison is drawn between our data and European findings on the work ability of nurses, reported by Camerino et al. [7], a different picture emerges. At age 45-49, Australian RNs achieve a level of work ability that compares favourably with the scores recorded in most of the 10 countries included in the Camerino et al. study (only the Netherlands and Norway achieved a higher average score in this age bracket). In the over-55 age group, only Finland, the Netherlands and Italy achieve higher average work ability scores than Australia.

Our finding that CCWs and PCAs achieve relatively high levels of work ability is also interesting when compared to the results of studies of work ability in other countries. For example, it contrasts the findings of Ilmarinen et al.'s [8] study of workers aged 45 and over, which showed that women in 'physically demanding' roles, such as 'home care' and 'auxiliary' roles had lower levels of work ability than women in 'mentally demanding' roles, such as nursing and teaching. Our finding challenges the notion that physically demanding work is, per se, a source of low work ability.

This study found that, among ENs and care workers, work ability was not significantly associated with age. Furthermore, although our results indicate that the work ability of RNs declines between age 45-49 and 50-54, work ability in this group stabilises after this age.

This contrasts the findings of some other studies and common stereotypes. Using longitudinal data, Ilmarinen et al. [8] found that generally the work ability of both mature age men and women declined with age. Camerino et al. [7] also found a statistically significant association between age and work ability (although their study was not limited to mature age workers).

Our findings of a generally weak relationship between age and work ability are more in line with Ilmarinen et al.'s [8] particular findings: changes in work ability among women engaged in mental work (including nursing) were not statistically significant; and there were no statistically significant associations between changes in work ability and age among women engaged in physical work in their 50s. Our findings (in combination with Ilmarinen et al.'s) suggest that older workers can sustain work ability. This may reflect the good health of many older workers, or their ability to find adaptations within their occupational roles. Regardless, the combined evidence recommends that managers and others exercise caution before assuming that older workers necessarily have low work ability.

On, perhaps, the key issue addressed in the study: the relationship between work ability and ITL; we find evidence of a strong negative correlation between the work ability of ENs, PCAs and CCWs, and ITL. The evidence assembled here suggests that ENs and care workers with low work ability tend to leave the aged care sector; and that high work ability is needed to remain. The finding implies that strategies aimed at retaining ENs, PCAs and CCWs in the sector will need to reflect the importance of work ability.

However, in contrast to the findings of Camerino et al. [7], we find that work ability is not strongly related to ITL for RNs. These differences potentially reflect the focus of our study on mature age RNs and/or our focus on the aged care sector. They suggest that factors other than work ability are more important in the determination of ITL for mature age RNs in Australia's aged care sector. These include the relatively low rate of pay on offer (compared with hospital and other settings) [11; p. 1]. RNs with high work ability might be more actively considering the prospect of working in another sector. However, the evidence that work ability is not strongly related to ITL for RNs could also indicate that RNs with low work ability perceive that moving to a nursing role other than aged care would be difficult or undesirable.

Given the strategic importance of mature age women workers in the aged care sector, further investigations of their work ability and employment retention are warranted. Possible extensions to the current study include examinations of the interrelationships between stress, low mood and poor workplace relationships on both work ability and ITL. 


\section{Key Points}

- The WAI measures workers' ability at present and in the near future to meet the demands of their jobs.

- This study suggests that mature Australian female aged care workers have relatively high levels of work ability.

- Furthermore, their work ability remains high in their $50 \mathrm{~s}$ and $60 \mathrm{~s}$, in contrast to some prevailing stereotypes.

- Work ability is a key determinant of ITL for ENs, PCAs and CCWs, suggesting that retention strategies incorporate programmes for occupational health and mental and physical wellbeing.

\section{References}

1 Brooke E, Goodall J, Handrus M, Mawren D. Applying workability in the Australian residential aged care context. Australasian Journal on Ageing 2013; 32: 130-134.

2 King D, Mavromaras K, Wei Z et al. The Aged Care Workforce, 2012. Canberra: Australian Government Department of Health and Ageing, 2012.
3 Productivity Commission. Caring for older Australians. Final Inquiry Report. Canberra; 2011 Report No. 53.

4 Austen S, Ong R. The effects of ill health and informal care roles on the employment retention of mid-life women: Does the workplace matter? Journal of Industrial Relations 2013; 55: 663-680.

5 Austen S, Jefferson T Intersections in Australian research: Older workers, women's labour supply and paid work in aged care. Refereed Proceedings of the 26th Annual Conference AIRAANZ, Gold Coast, 8-10 Feb 2012. 2012.

6 Shacklock K, Brunetto Y. The intention to continue nursing: Work variables affecting three nurse generations in Australia. Journal of Advanced Nursing 2011; 68: 36-46. doi: 10.1111/j.1365-2648.2011.05709.x.

7 Camerino D, Conway P, Van der Heijden B et al. Low-perceived work ability, ageing and intention to leave nursing: A comparison among 10 European countries. Journal of Advanced Nursing 2006; 56: 542552.

8 Ilmarinen J, Tuomi K, Klockars M. Changes in the work ability of active employees over an 11-year period. Scandinavian Journal of Work, Environment and Health 1997; 23 (Suppl. 1): 49-57.

9 van den Berg TIJ, Elders LAM, de Zwart BCH, Burdorf A. The effects of work-related and individual factors on the work ability index: A systematic review. Occupational and Environmental Medicine 2008; 66: 211-220. doi: 10.1136/oem.2008.039883.

10 Martin B, King D. Picture of the Residential and Community Based Aged Care Workforce 2007. Adelaide: National Institute of Labour Studies, Flinders University, 2008.

11 Nursing Career Allied Health. Aged care nurse and carer wages not good enough? 2013. [Cited 14 January 2014.] Available from URL: http:// www.ncah.com.au/news-events/aged-care-wages-cant-be-ignored/ $1856 /$. 